\title{
The crystal structure of rabbit IgG-Fc
}

\section{Enrico Girardi, Mary D. Holdom, Anna M. Davies, Brian J. Sutton and Andrew J. Beavil}

King's College London, Randall Division of Cell and Molecular Biophysics, London, UK MRC-Asthma UK Centre in Allergic Mechanisms of Asthma, London, UK New Hunt's House, Guy's Campus, London, SE1 1UL, UK

Short title: Rabbit IgG-Fc structure

\section{Keywords}

$\mathrm{IgG}, \mathrm{Fc}$ fragment, immunoglobulins, glycosylation, rabbit

\begin{abstract}
Abbreviations used:
IgG, immunoglobulin G; Fc, fragment crystallisable; Fab, antigen binding fragment; rmsd, root mean square deviation; MBP, mannose binding protein; RA, rheumatoid arthritis; RF, rheumatoid factor

\section{Address correspondence to:}

Brian Sutton, Randall Division of Cell and Molecular Biophysics, King's College London, New Hunt's House, Guy's Campus, London, SE1 1UL, UK. Tel: +44 (0) 207848 6423. E-mail brian.sutton@kcl.ac.uk
\end{abstract}




\begin{abstract}
We report the structure of the Fc fragment of rabbit IgG at 1.95 angstrom resolution. Rabbit IgG was the molecule for which Porter established the four-chain, Y-shaped structure of the antibody molecule, and crystals of the Fc ("Fragment crystallisable") were first reported almost 50 years ago in this journal (Porter R.R., 1959, Biochem. J., 73, 119-126). This high-resolution analysis, apparently of the same crystal form, reveals several features of IgG-Fc structure that have not previously been described. More of the lower hinge region is visible in this structure than in others, demonstrating not only the acute bend in the $\operatorname{IgG}$ molecule that this region can mediate, as seen in receptor complexes, but also that this region has a tendency to adopt a bent structure even in the absence of receptor. As observed in other IgG-Fc structures, the $\mathrm{C} \gamma 2$ domains display greater mobility/disorder within the crystals than the $\mathrm{C} \gamma 3$ domains; unexpectedly the structure reveals partial cleavage of both $\mathrm{C} \gamma 2$ intra-domain disulphide bonds, while an alternative conformation for one of the cysteine residues in the intact bridge within the more ordered $\mathrm{C} \gamma 3$ domains is observed. The N-linked oligosaccharide chains at Asn-297 are well-defined and reveal two alternative conformations for the galactose units on each of $\alpha(1-6)$ linked branches. The presence of this galactose unit is important for stabilising the structure of the entire branched carbohydrate chain, and its absence correlates with severity of autoimmune conditions such as rheumatoid arthritis in both human clinical studies and in a rabbit model of the disease. Rabbit IgG, through this highresolution structure of its $\mathrm{Fc}$ region, thus continues to offer new insights into antibody structure.
\end{abstract}




\section{Introduction}

The rabbit (Oryctolagus cuniculus) immunoglobulin gamma ( $\operatorname{IgG})$ was one of the first molecules of the immune system to be investigated, and the pioneering studies of Porter produced the very first information about the structure of the antibody molecule. Proteolytic cleavage of rabbit IgG by papain into three fragments, one of which crystallised [1] and was termed $\mathrm{Fc}$ (Fragment crystallisable), together with two identical fragments, termed Fab (Fragments antigen-binding), led to the first proposal of the four-chain, Y-shaped structure for IgG [2].

Even before the hetero-tetrameric model of two identical heavy and two identical light chains was developed, the first immunoglobulin allotypes were described for the rabbit $I G H G$ gene [3]. This observation led Oudin to hypothesize that IgG production was regulated by two independently segregating loci (named $a$ and $b$ ). Several years later these two loci were identified, respectively, in the variable region of the heavy chain and the constant region of the kappa light chain genes, confirming the presence of two distinct protein chains in the molecule.

Moreover, amino acid sequence studies on rabbit $\gamma$ chains were pivotal in clarifying the nature of immunoglobulin domains [4] and the arrangement of disulphide bonds present in the molecule [5]. Further enzymatic digestion and functional characterisation studies of rabbit IgG-Fc provided the first attempts to map the binding sites for complement and cytophilic activity [6-8]. Later, the same molecule was used as a model for the determination of the composition and topology of the carbohydrate chains present on the Fc fragment $[9,10]$, which play a role in stabilising the structure of the $\mathrm{Fc}$ and are required for binding to cell surface receptors.

Despite the key role played by this molecule in the history of immunology, no crystal structure is currently available for the $\mathrm{Fc}$ fragment of rabbit IgG. We describe here the $1.95 \AA$ resolution structure, one of the highest resolution Fc structures yet reported, from a crystal form that appears identical to the crystals pictured almost 50 years ago by Porter [1].

\section{Experimental}

\section{Protein purification}

Purified polyclonal rabbit IgG (Sigma-Aldrich, UK) was digested with papain according to the manufacturer's instructions (ImmunoPure Fab preparation kit, Pierce Corporation, USA). The Fab portion was removed from the resulting mixture using a protein A column. Fractions containing the $\mathrm{Fc}$ fragment and undigested $\mathrm{IgG}$ were then loaded onto a Superdex-200 gel filtration column (Amersham Pharmacia Biotech, UK) equilibrated with $0.5 \mathrm{M}$ Tris $\mathrm{pH} 7.2,0.25 \mathrm{M} \mathrm{NaCl}$ and $0.1 \%$ $(\mathrm{w} / \mathrm{v})$ sodium azide. The purity of the eluted sample was assessed by non-reducing SDS-PAGE; a single band indicated the presence of intact dimer (data not shown). Fractions containing the purified $\mathrm{Fc}$ were pooled and concentrated to $2 \mathrm{mg} / \mathrm{ml}$ at $4^{\circ} \mathrm{C}$ by centrifugation (Amicon, MWCO 10000, Millipore Corporation, USA) and stored at the same temperature until further use.

\section{Crystallisation of the Fc fragment}

Crystallisation conditions for the IgG-Fc fragment have previously been reported [8,9], but initial crystallisation trials using these conditions were unsuccessful. However, crystals were obtained in several conditions from a commercial sparse matrix screen (Crystal Screen I, Hampton Research, USA) and promising hits were further optimized using the hanging drop vapour diffusion method. Conditions that yielded crystals suitable for data collection were as follows: $1 \mathrm{ml}$ of $0.1 \mathrm{M}$ sodium 
acetate at $\mathrm{pH} 4.7$ and $2 \mathrm{M}$ sodium formate (reservoir) and $1 \mu$ protein solution with an equal volume of reservoir (drop). The drops were stored at $18^{\circ} \mathrm{C}$ and plate-like crystals measuring approximately $0.1 \mathrm{~mm}$ by $0.1 \mathrm{~mm}$ typically appeared after two days. Their morphology was identical to those pictured $[1,6]$ and characterised $[11,12]$ earlier.

\section{Data collection and processing}

Crystals were fished using nylon loops, soaked in a cryoprotectant solution ( $0.1 \mathrm{M}$ sodium acetate, $\mathrm{pH}$ 4.7, $2 \mathrm{M}$ sodium formate and 30\% glycerol) and flash-cooled in liquid nitrogen. Data were collected at beamline ID29 at the European Synchrotron Radiation Facility (ESRF, Grenoble, France). The data were processed with MOSFLM [13], SCALA [14] and the CCP4 program suite [15]. The space group and cell parameters were found to be very similar to those observed previously [12]. Calculation of the Matthews coefficient $(2.53 ; 51.1 \%$ solvent) indicated the presence of one Fc molecule per asymmetric unit. Data collection and statistics are presented in Table 1. The structure was solved by molecular replacement with MOLREP [15,16] using protein atoms from the human IgG-Fc structure as a search model (PDB code 1H3T [17]). Refinement was performed initially with REFMAC [18] and later with PHENIX [19]. TLS refinement was implemented using 10 TLS groups per chain, as suggested by the TLSMD server [20]. Cycles of refinement were alternated with rounds of manual model building with COOT [21]. Protein atoms were modelled first, followed by carbohydrate, ligands and water molecules. The refined structure was analysed with COOT, PROCHECK [22] and MolProbity [23]. Secondary structure was assigned with the KSDSSP algorithm implemented in Chimera [24]. Omit maps were prepared in CNS [25]. Figures were prepared with PyMOL (www.pymol.sourceforge.net). Refinement statistics are presented in Table 2.

Atomic coordinates and structure factors have been deposited in the PDB database with the accession code 2 VUO.

\section{Results and discussion}

\section{Overall structure}

The $1.95 \AA$ resolution structure of rabbit IgG-Fc displays the typical horseshoe-like arrangement of the $\mathrm{C} \gamma 2$ and $\mathrm{C} \gamma 3$ domains in the two polypeptide chains (Figure 1). Superimposition with the highest resolution human IgG-Fc structure (PDB code 1L6X; 73\% sequence identity with rabbit $\mathrm{IgG}-\mathrm{Fc}$ ), revealed RMSD values of 0.81 and $0.76 \AA$ for the two chains (performed with LSQMAN software [26]). Superimpositions of the individual domains (RMSD 0.37 and $0.47 \AA$ for $\mathrm{C} \gamma 3 ; 0.80$ and $0.85 \AA$ for $\mathrm{C} \gamma 2$ ) indicated that most of the differences between the rabbit and human structure occur in the $\mathrm{C} \gamma 2$ domains.

In the rabbit IgG-Fc structure, interpretable density was present for residues Pro-230 to Ser-444 in chain A, and residues Pro-231 to Ser-444 in chain B; the three C-terminal residues were disordered. At the N-termini, although no density was observed in either chain for Cys-229, the residue involved in the inter-chain disulphide bond, much more of the "lower hinge" region is visible here than in other Fc structures (usually defined from Pro-238), except those complexed with receptors (FcyR) that bind to this part of the Fc molecule. However, Pro-230 and Pro-231 (in chains A and B respectively) are only $7.8 \AA$ apart (distance between closest atoms), consistent with an intact interchain disulphide bridge between residues Cys-229. In Figure 1, this lower hinge region of the polypeptide chain points towards the viewer, and demonstrates the flexibility and acute bend in the $\mathrm{IgG}$ molecule that it can mediate. It is striking that the conformation of this region in rabbit $\mathrm{Fc}$ is very similar to that observed in Fc-receptor complexes (PDB codes 1E4K, 1T89, 1T83) [27,28] and 
in intact immunoglobulins (1HZH, 1IGT) [29, 30]. In the receptor complexes, a bend in this lower hinge region is demanded by the presence of the receptor. In the rabbit Fc structure, a single hydrogen bond between Glu-233 (chain B) and Arg-443 in the C $\gamma 3$ domain of a symmetry-related molecule may assist in stabilising this part of the structure, but the only other crystal packing contacts with any lower hinge region residues on either chain are a total of five van der Waals interactions (Glu-233 on both chains and Leu-235 on chain A; cut-off $<3.5 \AA$ ). Thus although the presence of a symmetry-related molecule in this crystal form may restrict the conformational freedom of the lower hinge compared with other Fc crystal structures, it appears that this part of the hinge has a natural tendency to adopt this "bent" conformation even in the absence of bound receptor.

The $\mathrm{C} \gamma 2$ domains are more mobile (or disordered) than the $\mathrm{C} \gamma 3$ dimer, as reflected by their higher overall B factors (average values 27.8 and $21.2 \AA^{2}$ respectively, Table 2). This feature has been seen in almost all other Fc structures, from the first reported human IgG-Fc [31] to the recent highresolution structure 1L6X (average values 23.8 and $18.7 \AA^{2}$ respectively). No significant difference in the $\mathrm{B}$ values was observed between the two chains of rabbit IgG-Fc however, implying that the values for $\mathrm{C} \gamma 2$ and $\mathrm{C} \gamma 3$ reflect an intrinsic difference between the two domains, and are not the result of different crystal packing environments.

The conformation of the main chain for residues 283-296, adjacent to residue Asn-297 to which carbohydrate is covalently attached, has been found to adopt one of two different conformations in other IgG-Fc structures; these have been termed the 'classical' and the 'complex' conformation [32]. In rabbit IgG-Fc, the structure conforms to the 'complex' conformation observed in the recently published high-resolution human IgG-Fc structures [17,32].

\section{Intra-domain disulphide bonds}

Each domain contains a conserved intra-chain disulphide bond, bridging the two $\beta$-sheets of the immunoglobulin fold. In both $\mathrm{C} \gamma 2$ domains, the electron density indicates the presence of both an intact covalent bond and a broken state for the Cys-261 to Cys-321 bridge (Figure 2A). The differences in conformation between the two cysteinyl residues derives principally from a rotation around the $\chi_{1}(\mathrm{~N}-\mathrm{C} \alpha-\mathrm{C} \beta-\mathrm{C} \gamma)$ torsion angle, and are almost certainly the result of radiation damage during data collection. In the $\mathrm{C} \gamma 3$ domains there is no evidence of intra-domain disulphide bond cleavage, but Cys-425 in chain A (Figure 2B) displays two alternative conformations, indicating that some degree of flexibility is present even in the core of this immunoglobulin domain. The partial cleavage of both bonds within the $\mathrm{C} \gamma 2$ domains is clearly consistent with the higher $\mathrm{B}$ values observed for this domain, as discussed above.

\section{Allelic variation}

Allotypes of the $I G H G$ gene, which codes for the only IgG heavy chain subclass in the rabbit, have been described in the constant region of the $\gamma$ heavy chain. They correlate with single amino-acid substitutions at positions 228 and 309 in the hinge and $C \gamma 2$ domain respectively [33]. Each locus presents two serologically defined alleles: $d 11$ and $d 12$ at position 228 and $e 14$ and $e 15$ at position 309. The two allelic variants at position 309, which is defined in the crystal structure, correspond to an Ala/Thr substitution. Given the nature of the rabbit IgG sample, no information was available regarding the sequence or the allelic proportions present in the crystallised protein. As the allelic variant with alanine is the more widespread [34], this residue (corresponding to the $e 15$ allotype) was refined at position 309. No positive density in the $\mathrm{F}_{\mathrm{o}}-\mathrm{F}_{\mathrm{c}}$ map (even at a contour level of $\sim 2.0 \sigma$ ) was observed, suggesting that the allele with Ala was the one exclusively present in the crystal. The 
presence of an Ala residue at position 309 was later confirmed by mass spectrometry analysis (data not shown). An allotypic variant (nG4m; Leu/Val) is also found at position 309 in human $\mathrm{IgG} 4$ [35]. This amino acid is located at the $\mathrm{C} \gamma 2 / \mathrm{C} \gamma 3$ interface, in the binding site for the neonatal $\mathrm{Fc}$ receptor [36]. While the two variants characterised in human $\mathrm{Fc}$ both code for hydrophobic residues, the $e 14$ allotype in rabbit $\mathrm{Fc}$ codes for a polar residue, which may affect the binding of the neonatal receptor, although there are no reported functional studies that test this hypothesis. Incidentally, we observed positive density on both chains at Ala-396 indicative of a threonine or valine residue, although no variability has been noted previously at this position.

\section{Glycosylation}

Rabbit IgG-Fc possesses a conserved glycosylation site at Asn-297 in each C $\gamma 2$ domain, at which a complex biantennary type oligosaccharide is covalently attached. The carbohydrate covers a hydrophobic patch on the $\mathrm{C} \gamma 2$ domain surface and extends into the cavity between the two $\mathrm{C} \gamma 2$ domains (Figure 3). Its presence stabilises and maintains the structure of the protein, as proposed initially from studies of the human IgG-Fc fragment [31] and later confirmed by microcalorimetry experiments [37,38] and structural studies of sequentially deglycosylated Fc glycoforms [17]. Moreover, the conserved oligosaccharide plays a crucial role in IgG effector functions: it is required for optimal Fc $\gamma$ receptor binding, and mediates interactions with serum lectins such as mannose binding protein (MBP) that leads to complement activation $[39,40]$.

While the core of the oligosaccharide is conserved, heterogeneity is observed in the fucosylation (found in $\sim 30-40 \%$ of rabbit IgG-Fc molecules), galactosylation ( $50-60 \%)$ and sialylation of the chain ( $25 \%)$; an additional bisecting $\mathrm{N}$-acetylglucosamine may also be present ( $25-30 \%$ of rabbit IgG-Fc molecules) $[41,42]$. On both chains, carbohydrate units up to and including the galactose at the end of each of the $\alpha(1-6)$ branches (Gal-6) were built into good electron density (Figure 3), whereas on the $\alpha(1-3)$ branches, only Man-7 and GlcNAc-8 (the latter only on chain C, Figure 3) were built. The lack of interpretable electron density for fucose, terminal sialic acid or a bisecting $\mathrm{N}$-acetylglucosamine is consistent with the low fractional compositions reported. The $\alpha(1-3)$ branches extend into the area between the two $C \gamma 2$ domains and lie close to each other, but none of the contacts between the two chains described previously in the much lower resolution analysis [9] were seen. While some unliganded human Fc structures show inter-chain carbohydrate contacts, others do not [43]; whether or not such contacts occur appears to depend upon domain quaternary structure, which in turn may be determined by crystal packing contacts. The $\alpha(1-6)$ branches lie close to the first two strands $\left(A\right.$ and $B$ ) of the $C_{\gamma} 2$ domain, their core regions interacting with residues Phe-241, Phe-243 and Glu-265.

The extent of galactosylation in $\mathrm{IgG}$ molecules is highly variable, with a reported $40-50 \%$ of the molecules entirely lacking galactose in healthy rabbits [41,42]. We observed density at the end of each $\alpha(1-6)$ branch, clearly indicating the presence of a galactose residue (Gal-6). When refined with an occupancy of $100 \%$ (as for all the other carbohydrate units), higher B factors and some negative difference electron density indicated a slightly lower occupancy and/or the presence of alternative conformations. (Refinement with occupancy values set between 40 and $60 \%$ revealed positive difference electron density). It was clear however, that the principal conformation adopted by Gal-6 is different in the two chains (Figures 1 and 3). In chain A, the $\mathrm{O} 6$ atom of Gal-6 points away from the $\mathrm{C} \gamma 2$ domain, while in chain $\mathrm{B}$, the $\mathrm{O} 6$ atom points towards the $\mathrm{C} \gamma 2$ surface. These two conformations are related by an approximately $180^{\circ}$ rotation of the plane of the hexose ring, but in both, Gal-6 lies in the same cavity, forming (different) hydrogen bonds to the same residues Pro244, Lys-246 (although density for CE and CZ of Lys-246 on chain A is ill-defined), Glu-258 and Thr-260. The galactose conformation in chain A is identical to that seen in the $1.65 \AA$ human IgG-Fc (PDB code 1L6X), but no asymmetry was observed in that structure as the two chains are related by 
a crystallographic dyad. There is thus some variability in the nature of the interaction between this carbohydrate unit and the protein surface.

\section{Fc galactosylation and functional implications}

The galactose content of human IgG-Fc has been shown to correlate inversely with disease progression in rheumatoid arthritis (RA) [44,45] and other auto-immune diseases [46]. Various mechanisms have been proposed: the absence of galactose may expose hydrophobic surfaces that promote Fc-mediated IgG aggregation, or generate new Fc epitopes that are recognised by rheumatoid factor (RF) autoantibodies [47]. The galactose unit is also known to be a key determinant for stabilising the carbohydrate chain's interaction with the protein domain. Its absence leads to greater mobility of the chains as shown by NMR [48] and crystallographic [49] studies, and this enhanced accessibility permits interaction with lectins such as MBP, leading to complement activation [40]. Similar changes in the galactose content of rabbit IgG have been reported after hyperimmunisation, and enhanced avidity of rabbit IgG RF autoantibodies for IgG-Fc was shown to be due to decreased galactose content [50]. In a rabbit model of RA, a long-term immunisation study that followed RF titre, avidity and IgG-Fc galactose content during disease progression showed the same inverse correlation between RF avidity for IgG-Fc and galactose content [51]. The crystal structure of rabbit $\mathrm{IgG}-\mathrm{Fc}$ has revealed a conformational variability in the mode of interaction of this key carbohydrate residue and the $\mathrm{C} \gamma 2$ domain, underlining the tenuous nature of this contact and its role in tipping the balance between a mobile carbohydrate chain and an ordered structure in contact with the $\mathrm{C} \gamma 2$ domain surface.

Rabbit IgG provided the very first insights into antibody structure, and through the high-resolution crystal structure of its $\mathrm{Fc}$ region reported here, it continues to reveal new structural details and enhance our understanding of the antibody molecule.

\section{Acknowledgements}

We are grateful to Sophia Karagiannis for assistance with the Fc fragment preparation and to Roberto Steiner, Stella Fabiane, Balvinder Dhaliwal and Silviana Comsa for helpful discussions. We are also grateful to Karen Homer (Dental Institute, King's College London) for the mass spectrometry analysis. We thank the staff at ID29 at ESRF (Grenoble, France) for assistance with data collection. E.G. is funded by a King's College London Strategic Development Studentship. 


\section{References}

1 Porter, R. R. (1959) The hydrolysis of rabbit gamma-globulin and antibodies with crystalline papain. Biochem. J. 73, 119-126

2 Fleischman, J. B., Porter, R. R. and Press, E. M. (1963) The arrangement of the peptide chains in gamma-globulin. Biochem. J. 88, 220-228

3 Oudin, J. (1956) The allotype of certain blood protein antigens. C. R. Hebd. Seances. Acad. Sci. 242, 2606-2608

4 Hill, R. L., Delaney, R., Lebovitz, H. E. and Fellows, Jr, R. E., (1966) Studies on the amino acid sequence of heavy chains from rabbit immunoglobulin G. Proc. R. Soc. Lond. B. Biol. Sci. 166, $159-175$

5 O'Donnell, I. J., Frangione, B. and Porter, R. R. (1970) The disulphide bonds of the heavy chain of rabbit immunoglobulin G. Biochem. J. 116, 261-268

6 Utsumi, S. (1969) Stepwise cleavage of rabbit immunoglobulin G by papain and isolation of four types of biologically active Fc fragments. Biochem. J. 112, 343-355

7 Colomb, M. and Porter, R. R. (1975) Characterization of a plasmin-digest fragment of rabbit immunoglobulin gamma that binds antigen and complement. Biochem. J. 145, 177-183

8 Ovary, Z., Saluk, P. H., Quijada, L. and Lamm, M. E. (1976) Biologic activities of rabbit immunoglobulin $\mathrm{G}$ in relation to domains of the Fc region. J. Immunol. 116, 1265-1271

9 Sutton, B. J. and Phillips, D. C. (1983) The three-dimensional structure of the carbohydrate within the Fc fragment of immunoglobulin G. Biochem. Soc. Trans. 11, 130-132

10 Rademacher, T. W., Homans, S. W., Fernandes, D. L., Dwek, R. A., Mizuochi, T., Taniguchi, T. and Kobata, A. (1983) Structural and conformational analysis of immunoglobulin-derived Nlinked oligosaccharides. Biochem. Soc. Trans. 11, 132-134

11 Poljak, R. J. and Dintzis, H. M. (1966) Papain fragmentation of rabbit gamma-g-globulin: A crystallographic study. J. Mol. Biol. 17, 546-547

12 Aschaffenburg, R., Lewis, M., Phillips, D. C., Press, E. M., Smith, S. G., Sutton, B. J. and Mountford, C. W. (1979) Crystallographic studies of immunoglobulins: Crystallization of the Fc fragment of rabbit IgG with and without cleavage of the inter-chain disulphide bridge. J. Mol. Biol. 135, 1033-1036

13 Leslie, A. G. W. (1992) Recent changes to the MOSFLM package for processing film and image plate data. Joint CCP4 + ESF-EAMCB Newsletter on Protein Crystallography No. 26.

14 Evans, P. R. (1997) Scaling of MAD data. Proceedings of CCP4 Study Weekend.

15 Collaborative Computational Project, Number 4. (1994) The CCP4 suite: Programs for protein crystallography. Acta Crystallogr. D Biol. Crystallogr. 50, 760-763

16 Vagin, A. A. and Teplyakov, A. (1997) MOLREP: An automated program for molecular replacement. J. Appl. Cryst. 30, 1022-1025 
17 Krapp, S., Mimura, Y., Jefferis, R., Huber, R. and Sondermann, P. (2003) Structural analysis of human IgG-Fc glycoforms reveals a correlation between glycosylation and structural integrity. J. Mol. Biol. 325, 979-989

18 Murshudov, G. N., Vagin, A. A. and Dodson, E. J. (1997) Refinement of macromolecular structures by the maximum-likelihood method. Acta Crystallogr. D Biol. Crystallogr. 53, 240-255

19 Adams, P. D., Grosse-Kunstleve, R. W., Hung, L. W., Ioerger, T. R., McCoy, A. J., Moriarty, N. W., Read, R. J., Sacchettini, J. C., Sauter, N. K. and Terwilliger, T. C. (2002) PHENIX: Building new software for automated crystallographic structure determination. Acta Crystallogr. D Biol. Crystallogr. 58, 1948-1954

20 Painter, J. and Merritt, E. A. (2006) Optimal description of a protein structure in terms of multiple groups undergoing TLS motion. Acta Crystallogr. D Biol. Crystallogr. 62, 439-450

21 Emsley, P. and Cowtan, K. (2004) Coot: Model-building tools for molecular graphics. Acta Crystallogr. D Biol. Crystallogr. 60, 2126-2132

22 Laskowsky, R. A., MacArthur, M. W., Moss, D. S. and Thornton, J. M. (1993) PROCHECK: A program to check the stereochemical quality of protein structures. J. Appl. Cryst. 26, 283-291

23 Davis, I. W., Leaver-Fay, A., Chen, V. B., Block, J. N., Kapral, G. J., Wang, X., Murray, L. W., Arendall, 3rd, W. B., Snoeyink, J., Richardson, J. S. and Richardson, D. C. (2007) MolProbity: All-atom contacts and structure validation for proteins and nucleic acids. Nucleic Acids Res. 35, W375-83

24 Pettersen, E. F., Goddard, T. D., Huang, C. C., Couch, G. S., Greenblatt, D. M., Meng, E. C. and Ferrin, T. E. (2004) UCSF chimera - a visualization system for exploratory research and analysis. J. Comput. Chem. 25, 1605-1612

25 Brunger AT, Adams PD, Clore GM, DeLano WL, Gros P, Grosse-Kunstleve RW, Jiang JS, Kuszewski J, Nilges M, Pannu NS, Read RJ, Rice LM, Simonson T, \& Warren GL (1998) Crystallography \& NMR system: A new software suite for macromolecular structure determination. Acta Crystallogr. D Biol. Crystallogr. 54, 905-921

26 Kleywegt, G. J. (1996) Use of non-crystallographic symmetry in protein structure refinement. Acta Crystallogr. D Biol. Crystallogr. 52, 842-857

27 Sondermann, P., Huber, R., Oosthuizen, V. and Jacob, U. (2000) The 3.2- $\AA$ crystal structure of the human IgG1 Fc fragment-Fc gamma RIII complex. Nature 406, 267-273

28 Radaev, S., Motyka, S., Fridman, W. H., Sautes-Fridman, C. and Sun, P. D. (2001) The structure of a human type III Fc gamma receptor in complex with Fc. J. Biol. Chem. 276, 1646916477

29 Saphire, E. O., Parren, P. W., Pantophlet, R., Zwick, M. B., Morris, G. M., Rudd, P. M., Dwek, R. A., Stanfield, R. L., Burton, D. R. and Wilson, I. A. (2001) Crystal structure of a neutralizing human IGG against HIV-1: A template for vaccine design. Science. 293, 1155-1159

30 Harris, L. J., Larson, S. B., Hasel, K. W., Day, J., Greenwood, A. and McPherson, A. (1992) The three-dimensional structure of an intact monoclonal antibody for canine lymphoma. Nature 360, 369-372 
31 Deisenhofer, J. (1981) Crystallographic refinement and atomic models of a human Fc fragment and its complex with fragment B of protein A from Staphylococcus aureus at 2.9- and $2.8-\AA$ resolution. Biochemistry 20, 2361-2370

32 Matsumiya, S., Yamaguchi, Y., Saito, J., Nagano, M., Sasakawa, H., Otaki, S., Satoh, M., Shitara, K. and Kato, K. (2007) Structural comparison of fucosylated and nonfucosylated Fc fragments of human immunoglobulin G1. J. Mol. Biol. 368, 767-779

33 Esteves, P. J., Alves, P. C., Ferrand, N. and van der Loo, W. (2002) Hotspot variation at the CH2-CH3 interface of leporid IgG antibodies (Oryctolagus, Sylvilagus and Lepus). Eur. J. Immunogenet. 29, 529-535

34 Esteves, P. J., Alves, P. C., Ferrand, N. and van der Loo, W. (2002) Restriction fragment alleles of the rabbit $I G H G$ genes with reference to the rabbit $I G H G C H 2$ or e locus polymorphism. Anim. Genet. 33, 309-311

35 Brusco, A., Saviozzi, S., Cinque, F., DeMarchi, M., Boccazzi, C., de Lange, G., van Leeuwen, A. M. and Carbonara, A. O. (1998) Molecular characterization of immunoglobulin G4 gene isoallotypes. Eur. J. Immunogenet. 25, 349-355

36 West, Jr, A. P., and Bjorkman, P. J. (2000) Crystal structure and immunoglobulin G binding properties of the human major histocompatibility complex-related $\mathrm{Fc}$ receptor. Biochemistry. 39, 9698-9708

37 Ghirlando, R., Lund, J., Goodall, M. and Jefferis, R. (1999) Glycosylation of human IgG-Fc: Influences on structure revealed by differential scanning micro-calorimetry. Immunol. Lett. 68, 4752

38 Mimura, Y., Church, S., Ghirlando, R., Ashton, P. R., Dong, S., Goodall, M., Lund, J. and Jefferis, R. (2000) The influence of glycosylation on the thermal stability and effector function expression of human IgG1-Fc: Properties of a series of truncated glycoforms. Mol. Immunol. 37, 697-706

39 Mimura, Y., Sondermann, P., Ghirlando, R., Lund, J., Young, S. P., Goodall, M. and Jefferis, R. (2001) Role of oligosaccharide residues of IgG1-Fc in Fc gamma RIIb binding. J. Biol. Chem. 276, 45539-45547

40 Malhotra, R., Wormald, M. R., Rudd, P. M., Fischer, P. B., Dwek, R. A. and Sim, R. B. (1995) Glycosylation changes of $\mathrm{IgG}$ associated with rheumatoid arthritis can activate complement via the mannose-binding protein. Nat. Med. 1, 237-243

41 Hamako, J., Matsui, T., Ozeki, Y., Mizuochi, T. and Titani, K. (1993) Comparative studies of asparagine-linked sugar chains of immunoglobulin $\mathrm{G}$ from eleven mammalian species. Comp. Biochem. Physiol. B 106, 949-954

42 Raju, T. S., Briggs, J. B., Borge, S. M. and Jones, A. J. (2000) Species-specific variation in glycosylation of IgG: Evidence for the species-specific sialylation and branch-specific galactosylation and importance for engineering recombinant glycoprotein therapeutics. Glycobiology 10, 477-486

43 Oganesyan, V., Damschroder, M. M., Leach, W., Wu, H. and Dall'Acqua, W. F. (2008) Structural characterization of a mutated, ADCC-enhanced human Fc fragment. Mol. Immunol. 45, $1872-1882$ 
44 Parekh, R. B., Dwek, R. A., Sutton, B. J., Fernandes, D. L., Leung, A., Stanworth, D., Rademacher, T. W., Mizuochi, T., Taniguchi, T. and Matsuta, K. (1985) Association of rheumatoid arthritis and primary osteoarthritis with changes in the glycosylation pattern of total serum IgG. Nature 316, 452-457

45 Axford, J. S. (1999) Glycosylation and rheumatic disease. Biochim. Biophys. Acta 1455, 219229

46 Cremata, J. A., Sorell, L., Montesino, R., Garcia, R., Mata, M., Cabrera, G., Galvan, J. A., Garcia, G., Valdes, R. and Garrote, J. A. (2003) Hypogalactosylation of serum IgG in patients with coeliac disease. Clin. Exp. Immunol. 133, 422-429

47 Newkirk, M. M., Fournier, M. J. and Shiroky, J. (1995) Rheumatoid factor avidity in patients with rheumatoid arthritis: Identification of pathogenic RFs which correlate with disease parameters and with the gal(0) glycoform of IgG. J. Clin. Immunol. 15, 250-257

48 Wormald, M. R., Rudd, P. M., Harvey, D. J., Chang, S. C., Scragg, I. G. and Dwek, R. A. (1997) Variations in oligosaccharide-protein interactions in immunoglobulin G determine the sitespecific glycosylation profiles and modulate the dynamic motion of the Fc oligosaccharides. Biochemistry 36, 1370-1380

49 Corper, A. L., Sohi, M. K., Bonagura, V. R., Steinitz, M., Jefferis, R., Feinstein, A., Beale, D., Taussig, M. J. and Sutton, B. J. (1997) Structure of human IgM rheumatoid factor Fab bound to its autoantigen IgG Fc reveals a novel topology of antibody-antigen interaction. Nat. Struct. Biol. 4, 374-381

50 Murray, J. S. and Brown, J. C. (1990) Evidence that the Fc region of autologous rabbit IgG isolated before and after hyperimmunization is structurally different: Recognition by rheumatoid factor and monoclonal antibodies. Clin. Exp. Immunol. 81, 286-292

51 Ciric, D., Milosevic-Jovcic, N., Ilic, V. and Petrovic, S. (2005) A longitudinal study of the relationship between galactosylation degree of $\operatorname{IgG}$ and rheumatoid factor titer and avidity during long-term immunization of rabbits with BSA. Autoimmunity 38, 409-416 
Tables

Table 1: Data collection and processing statistics

\begin{tabular}{|l|c|}
\hline Beamline & ID29 \\
\hline Detector & ADSC Q315r \\
\hline Detector distance $(\mathrm{mm})$ & 230 \\
\hline$\lambda(\AA)$ & 0.976 \\
\hline Resolution limit $(\AA)$ & 1.95 \\
\hline Space group & $P 2_{1}(4)$ \\
\hline Cell dimensions $\left(\AA{ }^{\circ}{ }^{\circ}\right)$ & $\begin{array}{c}\mathrm{a}=58.66, \mathrm{~b}=71.21, \mathrm{c}=69.03 \\
\alpha=\gamma=90.00, \beta=104.78\end{array}$ \\
\hline Unique reflections $^{\circ}$ & 40194 \\
\hline Outer shell $(\AA)$ & $2.00-1.95$ \\
\hline Completeness $(\%):$ overall (outer shell) & $99.9(99.9)$ \\
\hline Multiplicity: overall (outer shell) & $7.3(7.2)$ \\
\hline $\mathrm{I} / \sigma(\mathrm{I}):$ overall $($ outer shell) & $17.2(3.6)$ \\
\hline$R_{\text {merge }}$ overall (outer shell) & $0.107(0.449)$ \\
\hline
\end{tabular}

Table 2: Refinement statistics

\begin{tabular}{|c|c|}
\hline Resolution range $(\AA)$ & $56.7-1.95$ \\
\hline No. of: protein atoms & 3479 \\
\hline carbohydrate atoms & 186 \\
\hline water molecules & 398 \\
\hline formate ions & 6 \\
\hline azide ions & 2 \\
\hline glycerol molecules & 4 \\
\hline Average B factors $\left(\AA^{2}\right)$ & \\
\hline protein & 24.6 \\
\hline carbohydrate & 42.5 \\
\hline water molecules & 35.1 \\
\hline formate ions & 39.9 \\
\hline azide ions & 38.3 \\
\hline glycerol molecules & 48.5 \\
\hline protein $\mathrm{C} \gamma 2$ (chain $\mathrm{A})$ & 28.5 \\
\hline protein $\mathrm{C} \gamma($ chain $\mathrm{B})$ & 27.0 \\
\hline protein $\mathrm{C} \gamma 3$ (chain $\mathrm{A})$ & 21.8 \\
\hline protein $\mathrm{C} \gamma 3$ (chain $\mathrm{B})$ & 20.5 \\
\hline$R_{\text {cryst }}(\%)$ & 16.9 \\
\hline$R_{\text {free }}(\%)(5 \%$ of reflections) & 20.4 \\
\hline rmsd bond lengths $(\AA)$ & 0.004 \\
\hline rmsd bond angles $\left({ }^{\circ}\right)$ & 0.928 \\
\hline Ramachandran plot $(\%$ of total & favoured: 99.5, allowed: 0.5, \\
residues) & disallowed: 0 \\
\hline
\end{tabular}




\section{Figure legends}

\section{Figure 1 Overall structure of rabbit IgG-Fc}

In this stereo representation of the structure, the two polypeptide chains are shown in green and blue, with their oligosaccharide chains (indicated as $\mathrm{C}$ and D) in yellow. The position of the $\mathrm{N}$ termini on both chains is indicated by the letter $\mathrm{N}$. The $\mathrm{N}$-terminal, lower hinge region in each chain points directly towards the reader.

\section{Figure 2 Conformations of the intra-domain disulphide bridges}

Stereo images of the region surrounding the intra-chain disulphide bridges in $\mathrm{C} \gamma 2$ (Figure 2A) and $\mathrm{C} \gamma 3$ (Figure 2B) of chain A. The electron density is calculated from a composite simulated annealing omit map, contoured at $1 \sigma$, generated omitting $4 \%$ of the structure per cycle. Carbon atoms are represented in green, oxygen in red, nitrogen in blue and sulphur atoms in orange. The highly conserved tryptophan residues (277 and 381) adjacent to the disulphide bonds can be seen towards the back of the image. In $\mathrm{C} \gamma 2$ (Figure 2A) the disulphide bond is partially broken, while in C $\gamma 3$ (Figure 2B), alternative conformations for the intact bridge are observed.

\section{Figure 3 The carbohydrate structure of rabbit IgG-Fc}

A) Electron density for the N-linked oligosaccharides at Asn-297 in each polypeptide chain, calculated from a composite simulated annealing omit map (contour level: $1 \sigma$ ), is shown together with a representation of the two $\mathrm{C} \gamma 2$ domains. B) Schematic representation of the complex biantennary oligosaccharide chain present in rabbit IgG-Fc. The residues modelled in the electron density are highlighted in light grey (GlcNAc-8 is only modelled on chain C). 

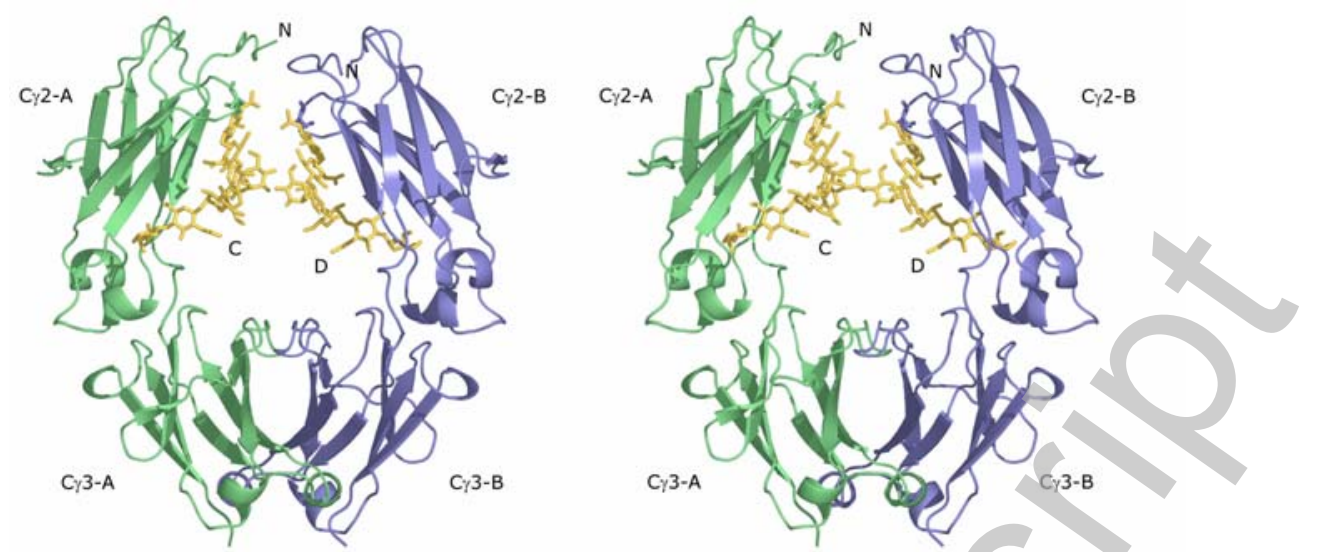

A

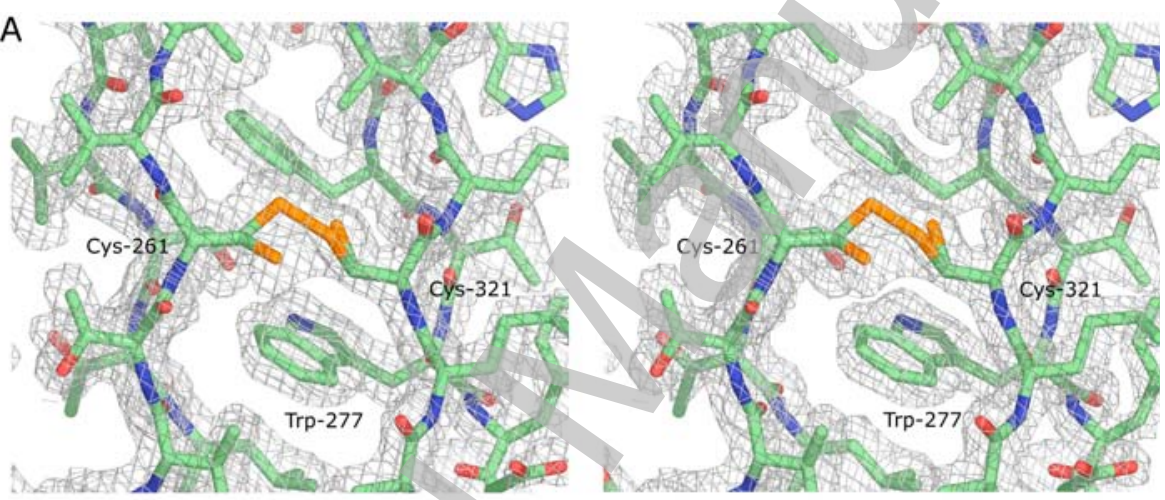

B
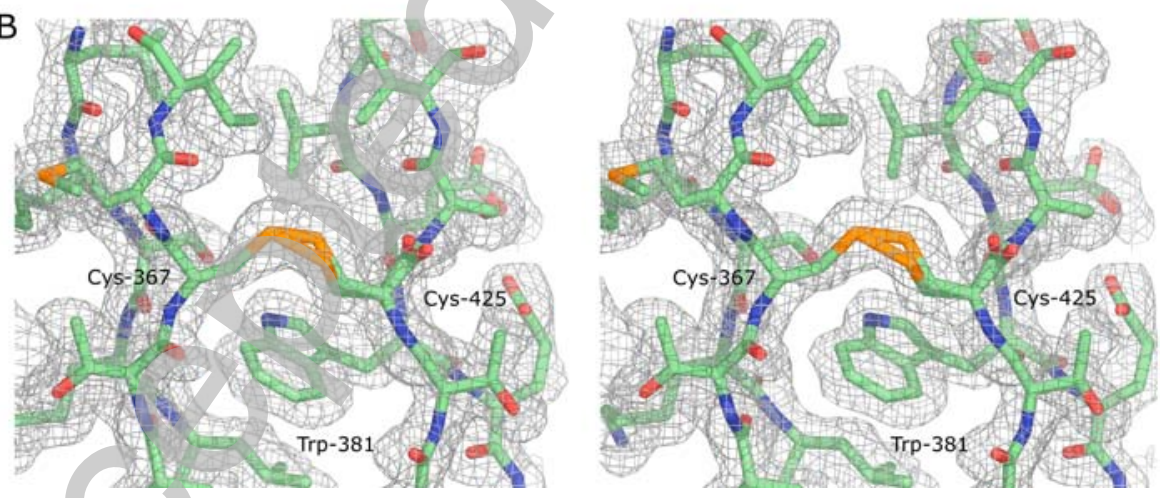
A

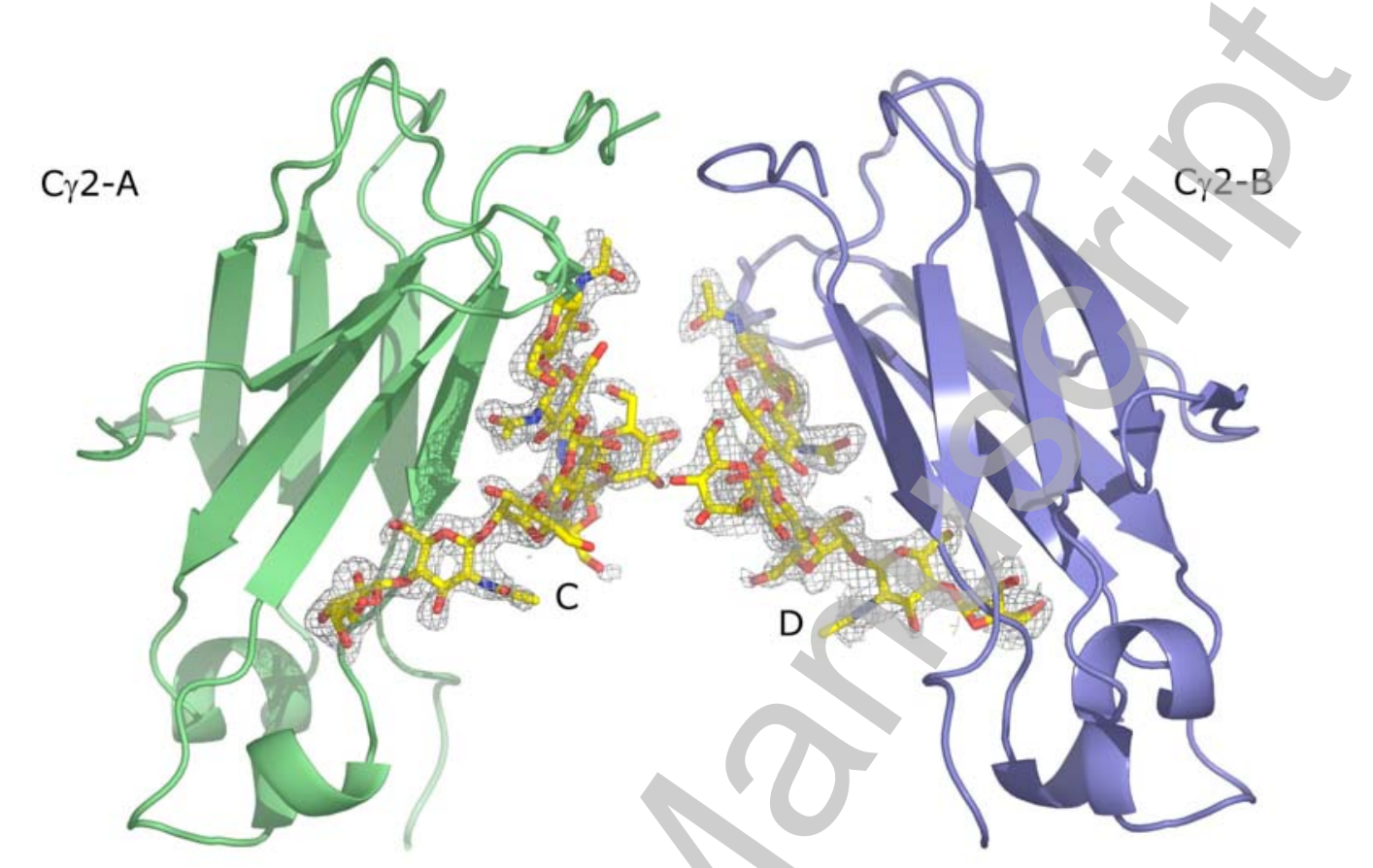

\section{B}

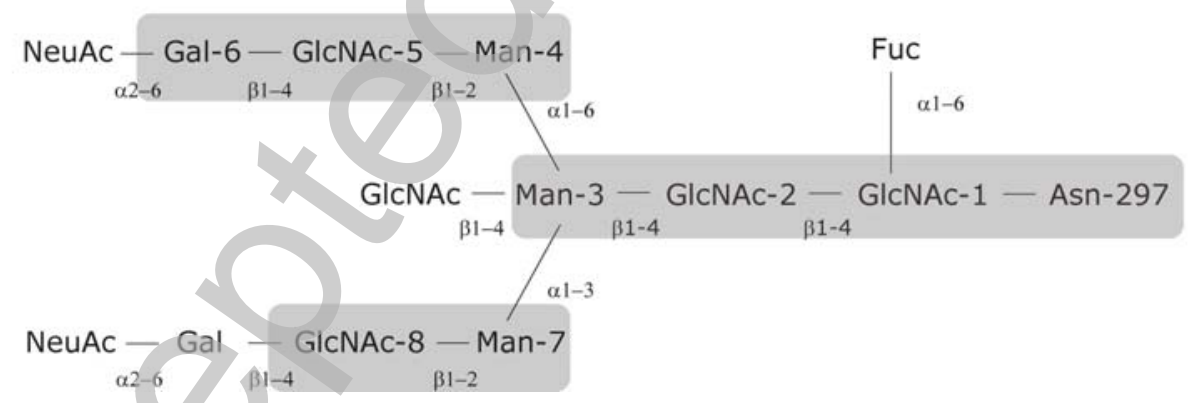

